\section{Review Article}

Check for updates

\title{
Tachycardia induced Cardiomyopathy
}

\section{OPEN ACCESS}

Received: Jun 28, 2019

Accepted: Jul 3, 2019

Correspondence to

Kyu-Hyung Ryu, MD, PhD

Division of Cardiology, Dongtan Sacred

Heart Hospital, Hallym University College

of Medicine, 7, Keunjaebong-gil, Hwaseong

18450, Korea.

E-mail: khryumd@hanmail.net

${ }^{*}$ Do Young Kim and Sung Hea Kim contributed equally to this work.

Copyright $@$ 2019. The Korean Society of Cardiology

This is an Open Access article distributed under the terms of the Creative Commons Attribution Non-Commercial License (https:// creativecommons.org/licenses/by-nc/4.0)

which permits unrestricted noncommercial use, distribution, and reproduction in any medium, provided the original work is properly cited.

ORCID iDs

Do Young Kim (D)

https://orcid.org/0000-0002-5601-9427

Sung Hea Kim (D)

https://orcid.org/0000-0001-5809-7958

Kyu-Hyung Ryu (iD)

https://orcid.org/0000-0001-9329-2716

\section{Conflict of Interest}

The authors have no financial conflicts of interest.

\section{Author Contributions}

Conceptualization: Ryu KH; Supervision: Ryu KH; Writing - original draft: Kim DY, Kim SH; Writing - review \& editing: Kim DY, Kim SH, Ryu $\mathrm{KH}$.

\author{
Do Young Kim (D, MD ${ }^{1, *}$, Sung Hea Kim (D, MD, PhD 2,*, and Kyu-Hyung Ryu (D, MD, PhD \\ 'Cardiovascular Center, Korea University Anam Hospital, Seoul, Korea \\ ${ }^{2}$ Division of Cardiology, Department of Medicine, Konkuk University Medical Center, Seoul, Korea \\ ${ }^{3}$ Department of Cardiology, Dongtan Sacred Heart Hospital, Hallym University College of Medicine, \\ Hwaseong, Korea
}

\begin{abstract}
Recent studies on radiofrequency catheter ablation (RFCA) in atrial fibrillation show its effectiveness in heart failure (HF) patients; hence, tachycardia-induced cardiomyopathy (T-CMP) is gaining attention. Tachycardia-mediated cardiomyopathy is a reversible left ventricular (LV) dysfunction, which can be induced by any tachyarrhythmia. Early recognition of T-CMP with appropriate treatment of the arrhythmia culprit will lead to the recovery of LV function. Patients with tachycardia and LV dysfunction should be suspected of having T-CMP, with or without established etiology of HF, because T-CMP may present by itself or contribute as a co-existent component. Therapeutic options include rate control, anti-arrhythmic drugs, or catheter ablation. Unlike in animal models, clinical data on human T-CMP is limited. Hence, future research should be more focused on tachyarrhythmia-induced cardiomyopathy as its burden is increasing.
\end{abstract}

Keywords: Heart failure; Tachycardia; Cardiomyopathies

\section{INTRODUCTION}

Long-standing tachycardia is a well-established cause of left ventricular (LV) systolic dysfunction. ${ }^{1-3)}$ Tachycardia-induced cardiomyopathy (T-CMP) can be defined as a heart failure (HF) syndrome originating from increased atrial or ventricular rates. ${ }^{4}$ Since the first description of T-CMP in the early 20th century, multiple studies have established a causerelationship between tachycardia and cardiomyopathy in both animal models and humans. ${ }^{5)}$ T-CMP is one of the major causes of congestive HF, which is a major health concern with high burden of morbidity. ${ }^{677} \mathrm{~T}$-CMP can be dramatically improved by appropriate management of tachycardia and HF. Thus, early recognition of T-CMP is important. ${ }^{1-4)}$ In this review, we will discuss the proposed pathophysiologic mechanism, diagnostic strategy, and treatment of T-CMP.

\section{CAUSES OF TACHYCARDIA-INDUCED CARDIOMYOPATHY}

T-CMP can be induced by various tachyarrhythmias, including atrial fibrillation (AF), incessant supraventricular tachycardias, and ventricular arrhythmias. ${ }^{5)}$ Although the 
incidence of T-CMP has not been investigated thoroughly in previous literature, T-CMP can develop as early as a week after the onset of tachycardia or it can take several years following the presentation of symptoms of tachycardia. ${ }^{3{ }^{38}} \mathrm{AF}$ with rapid ventricular response is the most common cause of T-CMP. ${ }^{3)}$ As AF facilitates development of HF regardless of rapid ventricular response and $\mathrm{HF}$ itself is predisposed to $\mathrm{AF},{ }^{910)}$ it is difficult to determine the exact proportion of T-CMP in these patients. However, earlier studies regarding rhythm control or rate control in patients with $\mathrm{AF}$ and systolic dysfunction suggest that the tachycardia-induced cardiomyopathy component is common in those patients. ${ }^{10-14)}$ Benefits of heart rate control were independent of atrioventricular synchrony and atrial contraction in those patients who received rate control with atrioventricular (AV) junction ablation and permanent pacemaker implantation. ${ }^{1213)}$ Studies investigating the time course of hemodynamic improvement following cardioversion in patients with AF and HF show that there is a time lag between restoration of sinus rhythm and LV systolic function, suggesting the presence of underlying myocardial failure. ${ }^{14-16)}$ Focal atrial tachycardia (AT) is a well-known cause of T-CMP. ${ }^{1517)}$ Although focal AT is usually benign, ${ }^{18}$ it can occasionally show an incessant nature and may cause T-CMP. In a series of focal AT patients who underwent catheter ablation, $10 \%$ of those patients presented with T-CMP had improved LV function following catheter ablation. ${ }^{17}$ It has been reported that T-CMP can be induced by atrioventricular nodal reentrant tachycardia (AVNRT), atrioventricular reciprocating tachycardia (AVRT), or permanent junctional reciprocating tachycardia (PJRT). ${ }^{19-23)}$ Given that reentrant supraventricular tachycardias (SVT), including AVNRT and AVRT, are mostly paroxysmal and rarely incessant, reentrant SVT induced T-CMP is uncommon. ${ }^{15)}$ Dual AV node physiology can facilitate T-CMP through dual atrioventricular nodal non-reentrant tachycardia, which is uncommon. ${ }^{1524)}$ Dual AV nodal non-reentrant tachycardia is the double firing of a single sinus beat which are conducted into both fast and slow pathways, simultaneously. If this phenomenon repeats rapidly, tachycardias can occur, and catheter ablation of the slow AV nodal pathway is curative in these patients. ${ }^{24)}$ Ventricular tachycardia (VT) is another cause of T-CMP. VTs causing T-CMP commonly originate from outflowing tracts or coronary cusps, ${ }^{15)}$ and idiopathic left ventricular tachycardia (ILVT) may also induce T-CMP. ${ }^{25)}$ There is a consensus that tachycardias of higher heart rate and longer duration could lead to cardiomyopathy (CMP). ${ }^{3)}$ However, the exact heart rate level that may induce T-CMP is not well defined. ${ }^{13)}$ Deterioration of left ventricular function could be induced at pacing rate of over 240 beats/min and 450 beats/min in the dog and rabbit model, respectively..$^{5)}$ Those pacing rates are approximately two times faster than normal heart rates of dogs (120 bpm) and rabbits (240 bpm). In our cohort of T-CMP, most of patients presented with heart rate of greater than $110-120$ beats/min. ${ }^{26)}$ Another clinical trials also have reported that patients with T-CMP had similar range of heart rate. ${ }^{1723) 27}$ In this context, the heart rate of 110-120 beats/min which is approximately two times faster than normal heart rate of human can be considered as a threshold of T-CMP.

There has been data regarding risk factors that contribute to the development of T-CMP. A study showed that patients with a polymorphic homozygous deletion in the angiotensinconverting enzyme (DD) gene may have a higher chance of developing T-CMP when persistent tachycardia is present. ${ }^{28)}$

The time lag from the diagnosis of tachyarrhythmia to the presentation of T-CMP was shorter among older patients with T-CMP as compared with younger patients with T-CMP in our cohort of T-CMP. ${ }^{26)}$ This may suggest aging is a factor that make patients more vulnerable to T-CMP. 


\section{PATHOPHYSIOLOGY}

In animal models, chronic rapid atrial or ventricular pacing results in low-output HF, which is similar to human HF, and the degree of ventricular dysfunction is related to the rate and duration of pacing. ${ }^{529}$ The cardiac changes involve LV systolic and diastolic dysfunction, a markedly dilated cardiomyopathy (DCMP) involving all cardiac chambers. ${ }^{\left.1{ }^{5}\right)}$ The structural changes of T-CMP are characterized by preservation or thinning of biventricular wall thickness without gross hypertrophy and increased ventricular mass. ${ }^{53031)}$ At a microscopic level, myocyte loss and elongation has been reported in T-CMP animal models. ${ }^{15 / 32 / 33)}$ Additionally, hypertrophic change of myocytes was documented in an animal model of T-CMP. ${ }^{33}$ However, there is controversy over hypertrophic change of myocytes because several studies have shown ventricular remodeling of T-CMP without evidence of myocyte hypertrophy. ${ }^{1531)}$ Reduction of the extracellular matrix and disruptions to the basement membrane-sarcolemmal interface were also observed in animal models. ${ }^{3134 / 35)}$ The extracellular matrix has a role in myocyte alignment and the mechanical ventricular performance is produced by interactions among myocytes, the basement membrane, and the extracellular matrix. ${ }^{534)}$ Consequently, these alternans lead to ventricular dilatation and contractile dysfunction. ${ }^{5 / 32 / 34)}$ Development of T-CMP induce alternans of myocyte electrophysiological characteristics which are accompanied by a reduced amplitude of action potential and peak L-type $\mathrm{Ca}^{2+}$ current and prolonged duration of the action potential. $\left.{ }^{36}\right)$ These changes also contribute to the myocyte contractile dysfunction with T-CMP. ${ }^{2366}$ In a study investigating isolated ventricular myocyte preparations from animal models with T-CMP, myocytes with T-CMP showed decreased density of T-tubules and L-type calcium channels which contribute to abnormal excitation-contraction coupling. ${ }^{37)}$ The hemodynamic changes of T-CMP involve elevated systemic resistance, elevated LV filling pressures, and increased LV wall stress. ${ }^{15)}$ Similar to other forms of HF, the neurohormonal system is upregulated in response to these changes in T-CMP.5)15) In similar HF models, induced by different causes, animals with T-CMP showed a blunted response to betaadrenergic stimulation with decreased beta1-receptors and alterations in beta-receptor transduction. ${ }^{529)}$ Blunted beta-adrenergic response is related to a decreased force-frequency relation on cardiac contractility ${ }^{29)}$ and this could aggravate impaired cardiac function during stress conditions in subjects with T-CMP. ${ }^{38}$ Depletion of myocardial energy stores have been proposed as a mechanism of T-CMP. Diminished levels of creatine, phosphocreatine, adenosine triphosphate and glycogen, enhanced activity of Krebs cycle oxidative enzymes, and decreased activity of the sodium-potassium adenosine triphosphatase (Na-K ATPase) pump were described in animals with T-CMP. ${ }^{5 / 35339}$ These metabolic changes are related to mitochondrial injury and impaired mitochondrial activity. ${ }^{15)}$ Increased levels of oxidative stress are associated with myocyte apoptosis, and mitochondrial DNA is more vulnerable to oxidative damage.4)15) Subclinical myocardial ischemia is another possible mechanism of T-CMP. Myocardial blood flow is decreased with significant remodeling of the coronary capillary vasculature. ${ }^{40)}$ Hibernation of myocardium due to ischemia may exist in T-CMP and this may partly explain the reversed remodeling of myocardium after cessation of the tachycardia. ${ }^{4)}$ Earlier changes of T-CMP would mainly be due to tachyarrhythmia. On the contrary, later pathologic changes of T-CMP are involved in neurohormonal changes following hemodynamic alternans. Therefore, pathophysiology of T-CMP shares common features with the pathophysiology of HF due to other etiologies. ${ }^{15)}$ A recent study investigated endomyocardial biopsy samples from patients with T-CMP and compared them with those from HFs due to other etiologies. In this study, electron microscopic examination revealed that T-CMP was characterized by hypertrophied cardiomyocytes, enhanced macrophage 
infiltration, and more severe disruption of mitochondria as that seen in idiopathic DCMP.41) This observation corresponds with the findings from animal models of T-CMP. Figure 1 summarized the pathophysiologic changes in T-CMP.

\section{CLINICAL PRESENTATION AND DIAGNOSIS}

The most common symptoms of T-CMP include palpitations, dyspnea, and presyncope/ syncope; although some patients with T-CMP may not present symptoms. ${ }^{27)}$ Development of T-CMP can take one month to years from the presentation of initial arrhythmia symptoms. ${ }^{3}$ In a series of T-CMP patients, T-CMP developed over 1 week after the onset of symptoms due to tachyarrhythmia. ${ }^{8}$ T-CMP should be suspected in all patients with newly diagnosed LV dysfunction without obvious etiologies and prior, persistent, or frequent paroxysmal tachycardia, because even severe LV dysfunction, so-called terminal LV dysfunction, can be normalized with appropriate treatment in T-CMP. ${ }^{42}$ Patients with underlying structural heart diseases and tachyarrhythmia should not be excluded because they may have a co-existent component of T-CMP. Previous normal LV function and LV dysfunction, disproportional to the degree of underlying heart disease, raise the possibility of T-CMP. ${ }^{42}$ The culprit tachycardia may not be documented at the time of presentation and underlying tachyarrhythmias such as AF or atrial flutter may not be the causative tachycardia. ${ }^{3 / 42}$ Therefore, monitoring for arrhythmia should be performed in patients with DCMP of unknown etiology or possible T-CMP and ambulatory electrocardiographic monitoring can be a useful tool for detecting the culprit tachyarrhythmia and for investigating the cause of the tachyarrhythmia in this setting. ${ }^{143}$ Evaluation of patients with a potential T-CMP includes common diagnostic strategies recommended by contemporary guidelines for HF.344445) Thyroid function tests should be included in the initial diagnostic test because hyperthyroidism can facilitate T-CMP with tachycardias such as AF or sinus tachycardia. ${ }^{3)}$ Plasma concentration of brain natriuretic peptides (BNPs) and NT pro-BNP are usually elevated in the setting of LV dysfunction due to $\mathrm{T}-\mathrm{CMP}^{1)}$ and rapid fall of plasma natriuretic peptide level following elimination of tachycardia suggests that $\mathrm{LV}$ dysfunction is due to T-CMP. ${ }^{46)} \mathrm{As} \mathrm{LV}$ wall thickness is usually preserved in T-CMP, absence of LV hypertrophy pattern on electrocardiography may suggest T-CMP as a cause of heart failure and can facilitate further imaging studies focusing on differential points of T-CMP. On echocardiography, T-CMP is characterized by dilated LV dimension with a lack of ventricular hypertrophy. ${ }^{15(543)}$ In a study comparing T-CMP and idiopathic DCMP, LV dimension and LV mass index were significantly smaller in the T-CMP group compared to the idiopathic DCMP group, and LV end-diastolic dimension $\leq 61 \mathrm{~mm}$ was a predictor of T-CMP. ${ }^{47)} \mathrm{In}$ addition to LV end-diastolic dimension, post-extra systolic potentiation of $\mathrm{LV}$ wall motion was more frequently observed in T-CMP rather than in DCMP from our previous work. ${ }^{26)}$

Cardiac magnetic resonance imaging (CMRI) can help differentiate T-CMP from idiopathic DCMP. In a study of patients with premature ventricular contractions-induced CMP or VTinduced CMP, absence of late gadolinium enhancement suggests an underlying scar in the $\mathrm{LV}$ wall which can be used as a predictor of T-CMP among patients with new onset DCMP of unknown etiology. ${ }^{4348)}$ During the early stages, T-CMP cannot easily be differentiated from $\mathrm{HF}$ of other etiologies and the final diagnosis of T-CMP can only be made by observation of improvement in LV systolic function. In case of improvement, recovery can be achieved within 1 to 6 months after appropriate treatment for tachycardia and HF.1) 
Baseline myocyte and extracellular components

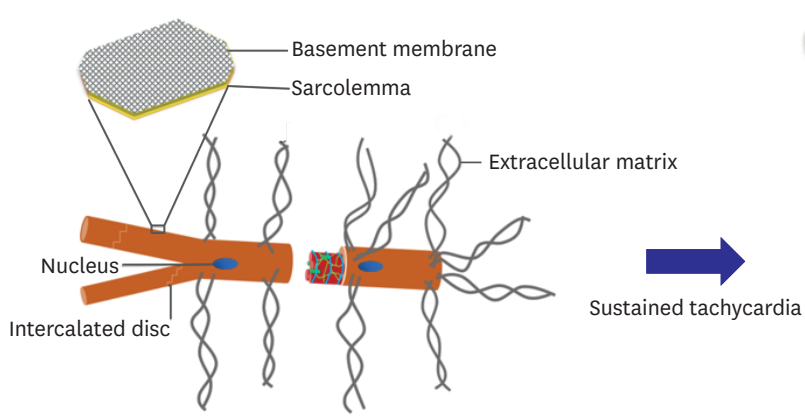

Cellular and extracellular changes of T-CMP

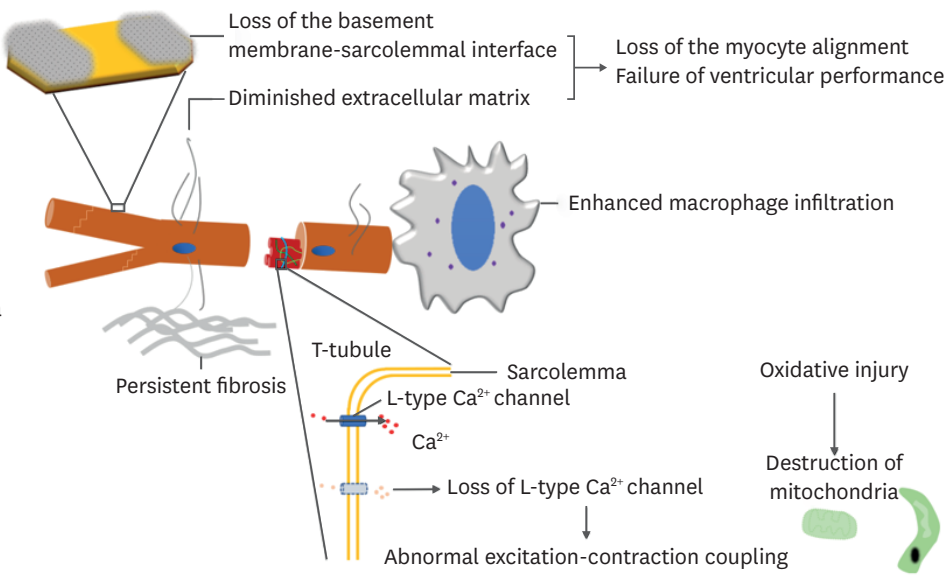

B
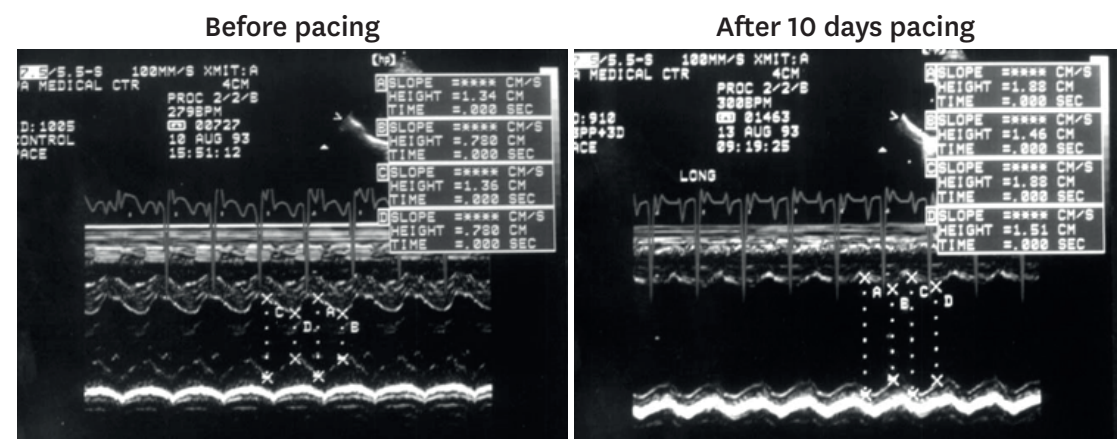

C

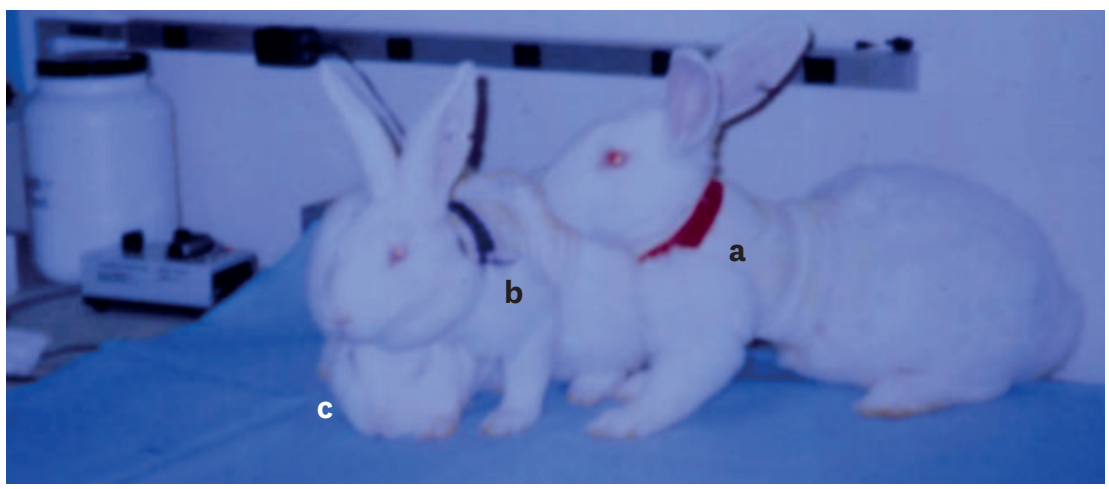

Figure 1. (A) Changes in cellular level in T-CMP. (B and C) Echocardiographic and gross change in pacing induced HF animal model. (A) Following sustained tachycardia, intracellular and extracellular remodeling leads to LV remodeling and worsening contractility. Decrease in L-type Ca ${ }^{2+}$ channel causes abnormal excitation-contraction coupling. Myocardial fibrosis persists even after recovery of LV function. (B) Echography in animal with pacing-induced HF (from Ryu et al.. ${ }^{29)}$ Ross et al. $\left.{ }^{38}\right)$ ). (C) Gross change in pacing-induced heart failure in animal (from Ryu et al. ${ }^{29}$ ), Ross et al..$\left.{ }^{38}\right)$ ): (a) normal rabbit before pacing, (b) low cardiac output status of rabbit after pacing with skin color change on ear and decreased activity, and (c) pacing induced HF rabbit showing edematous change and lethargic activity.

$\mathrm{HF}=$ heart failure; $\mathrm{LV}=$ left ventricular; $\mathrm{T}-\mathrm{CMP}=$ tachycardia-induced cardiomyopathy.

\section{TIME COURSE OF RECOVERY AND PROGNOSIS}

In animal models of T-CMP, recovery of cardiac systolic dysfunction after cessation of rapid pacing is a unique feature of this model. ${ }^{5} \mathrm{LV}$ systolic function improves within 1 to 2 weeks after the rapid pacing stops. Most of the hemodynamic variables return to normal by 4 weeks. ${ }^{5449}$ On the other hand, cessation of rapid pacing does not guarantee a complete recovery of the 
enlarged LV volume and LV diastolic dysfunction and LV hypertrophy did develop within 4 weeks after the cessation of rapid pacing in animal models. This phenomenon can be explained by compensatory remodeling following rapid pacing or by the inability of the hypertrophic changes in response to signals inducing hypertrophy during the pacing phase. ${ }^{515)}$

In clinical studies, improvement of LV systolic function was observed within a month following the treatment for tachyarrhythmia. ${ }^{22}$ Recovery of LV function can take 2-3 months and usually does not take longer than 6 months. ${ }^{126) 42}$ It is thought that the recovery of LV function can be shortened with adequate heart rate reduction treatment, below 100 beats/ min in our experience. A study reported that patients who had recovered from T-CMP had a significantly higher LV volume when compared to age-, sex-, and ejection fraction-matched controls..$^{50)}$ This result is consistent with the previous findings in animal models and suggests the persistence of negative $\mathrm{LV}$ remodeling in patients with T-CMP even after normalization of LV ejection fraction. ${ }^{115550)}$ Patients who recovered from T-CMP are vulnerable to the recurrence of tachycardia. ${ }^{1)}$ In cases where tachycardia reoccurred, deterioration of LV systolic function was induced more rapidly than that in previous T-CMP events ${ }^{851)}$ and persistence of microstructural changes following normalization of $\mathrm{LV}$ dysfunction seemed to be the cause of this vulnerability. ${ }^{50)}$ In this context, some studies reported sudden cardiac deaths after restoration of LV dysfunction due to T-CMP, and the prevalence of sudden cardiac death was not uncommon even after appropriate treatment and clinical improvement. ${ }^{8511}$

\section{TREATMENT}

Suppression of the culprit tachycardia is a key to the restoration of LV dysfunction for T-CMP. If T-CMP is suspected, treatment focusing on eliminating or controlling the culprit tachycardia should be pursued. ${ }^{133433}$ This approach includes radiofrequency catheter ablation (RFCA) of culprit arrhythmia or antiarrhythmic drugs. As discussed earlier, recurrence of arrhythmia has a deleterious effect on LV systolic function. RFCA should be considered as the first therapy for arrhythmias which can be managed with a high success rate such as atrial flutter, focal AT, AVNRT and AVRT. ${ }^{43}$

In case of decompensated $\mathrm{HF}$ with $\mathrm{AF}$, it is generally recommended to begin with a heart rate-control treatment because recurrence rate of $\mathrm{AF}$ following cardioversion is common. ${ }^{42)}$ Cardioversion should be performed following the acute management with optimal HF medication. ${ }^{42525}$ In our experience, digoxin is a preferred choice for the initial rate control after excluding coronary artery disease. After appropriate rate control, heart failure management and anticoagulation therapy, chemical cardioversion with amiodarone can be performed without a concern for procedure related complications and with a moderate success rate. ${ }^{11}$ Both rate and rhythm control strategies are reasonable choices for patients with $\mathrm{AF}$ with compensated $\mathrm{HF}{ }^{9{ }^{910)}}$ It has been proposed that lenient rate control is as effective and feasible as strict rate control in patient with $\mathrm{AF}^{53}{ }^{53}$ However, the RACE II trial included a limited number of patients with reduced LV systolic function, and more data is required to set up an ideal heart rate target for these patients. ${ }^{10) 43}$ Until then, a resting heart rate of up to 80 beats/min and up to 115 beats/min during moderate exercise should be an acceptable rate control target for AF patients with possible diagnosis of T-CMP.910)

Initial treatment of T-CMP should include standard HF therapy such as angiotensin-converting enzyme inhibitors or angiotensin receptor blockers, aldosterone blockers, beta-blockers, and 
diuretics to attenuate negative remodeling and relieve symptoms related to HF. ${ }^{13342-44)}$ Negative remodeling, including myocardial fibrosis and sustained LV volume enlargement is persistent even after normalization of LV systolic function. ${ }^{50154)}$ Hence, continuing standard HF management is advised for favorable remodeling. However, the duration for such therapy is not well defined. ${ }^{43}$

\section{FUTURE DIRECTION}

Since the first reported case of T-CMP in early 1900s, T-CMP has been recognized as a distinct disease entity and we have extended our knowledge on T-CMP with multiples studies of animal models and clinical trials. Animal models contributed to a better understanding of the pathophysiology by sustained tachycardias. However, there is limited data on the pathophysiology of T-CMP in humans. With development of cardiac imaging including CMRI, our knowledge on the pathophysiology of T-CMP from animal models will be translated into the clinical practice and lead to a better understating of the pathophysiology of T-CMP in humans. We still have limited data on the risk factors of T-CMP. Further studies on genetic and clinical characteristics guided risk prediction models for T-CMP are necessary for early detection of high-risk patients with tachyarrhythmias and to prevent development of HF. Recent AF ablation trials suggested that AF alone can lead to CMP independently of rapid ventricular response $\mathrm{e}^{55}$ and a more comprehensive term of AF induced CMP has been introduced. But there are gaps in our understanding of AF-induced CMP. Further studies are necessary to reveal the mechanism of AF induced CMP and to suggest appropriate treatment.

\section{CONCLUSION}

T-CMP is a reversible cause of HF. Imaging studies may be helpful for the diagnosis of T-CMP but diagnosing T-CMP requires the demonstration of improved LV function with suppression of tachycardia. T-CMP should be suspected in all patients with DCMP of undetermined etiology and tachycardia faster than 100 or 110 beats/min for early detection and treatment for these patients. T-CMP should be considered as a possible diagnosis even in patients with HF of other established causes because they may have the superimposed reversible component of T-CMP. Strategies of tachyarrhythmia management can be antiarrhythmic drugs, catheter ablation or rate control. Catheter ablation should be considered for tachyarrhythmias which can be curable with ablation. Recent studies on premature ventricular contractions-induced CMP and AF ablation trials in HF patients suggested arrhythmias besides T-CMP. In this regard, our focus should move from T-CMP to arrhythmia induced CMP.

\section{REFERENCES}

1. Huizar JF, Ellenbogen KA, Tan AY, Kaszala K. Arrhythmia-induced cardiomyopathy: JACC State-of-the-Art Review. J Am Coll Cardiol 2019;73:2328-44. PUBMED | CROSSREF

2. Martin CA, Lambiase PD. Pathophysiology, diagnosis and treatment of tachycardiomyopathy. Heart 2017;103:1543-52. PUBMED | CROSSREF

3. Bozkurt B, Colvin M, Cook J, et al. Current diagnostic and treatment strategies for specific dilated cardiomyopathies: a scientific statement from the American Heart Association. Circulation 2016;134:e579-646. PUBMED | CROSSREF 
4. Khasnis A, Jongnarangsin K, Abela G, Veerareddy S, Reddy V, Thakur R. Tachycardia-induced cardiomyopathy: a review of literature. Pacing Clin Electrophysiol 2005;28:710-21. PUBMED | CROSSREF

5. Shinbane JS, Wood MA, Jensen DN, Ellenbogen KA, Fitzpatrick AP, Scheinman MM. Tachycardia-induced cardiomyopathy: a review of animal models and clinical studies. J Am Coll Cardiol 1997;29:709-15. PUBMED | CROSSREF

6. Youn JC, Han S, Ryu KH. Temporal trends of hospitalized patients with heart failure in Korea. Korean Circ J 2017;47:16-24. PUBMED | CROSSREF

7. Schocken DD, Arrieta MI, Leaverton PE, Ross EA. Prevalence and mortality rate of congestive heart failure in the United States. J Am Coll Cardiol 1992;20:301-6. PUBMED | CROSSREF

8. Watanabe H, Okamura K, Chinushi M, et al. Clinical characteristics, treatment, and outcome of tachycardia induced cardiomyopathy. Int Heart J 2008;49:39-47. PUBMED | CROSSREF

9. Batul SA, Gopinathannair R. Atrial fibrillation in heart failure: a therapeutic challenge of our times. Korean Circ J2017;47:644-62. PUBMED | CROSSREF

10. Anter E, Jessup M, Callans DJ. Atrial fibrillation and heart failure: treatment considerations for a dual epidemic. Circulation 2009;119:2516-25. PUBMED | CROSSREF

11. Yom HJ, Park SH, Jeon SH, Chang JE. Efficacy of amiodarone in tachycardia induced cardiomyopathy due to non-valvular atrial fibrillation. Korean Circ J 2001;31:1305-10. CROSSREF

12. Redfield MM, Kay GN, Jenkins LS, Mianulli M, Jensen DN, Ellenbogen KA. Tachycardia-related cardiomyopathy: a common cause of ventricular dysfunction in patients with atrial fibrillation referred for atrioventricular ablation. Mayo Clin Proc 2000;75:790-5. PUBMED | CROSSREF

13. Edner M, Caidahl K, Bergfeldt L, Darpö B, Edvardsson N, Rosenqvist M. Prospective study of left ventricular function after radiofrequency ablation of atrioventricular junction in patients with atrial fibrillation. Br Heart J1995;74:261-7. PUBMED | CROSSREF

14. Lipkin DP, Frenneaux M, Stewart R, Joshi J, Lowe T, McKenna WJ. Delayed improvement in exercise capacity after cardioversion of atrial fibrillation to sinus rhythm. Br Heart J1988;59:572-7. PUBMED | CROSSREF

15. Ellis ER, Josephson ME. What about tachycardia-induced cardiomyopathy? Arrhythm Electrophysiol Rev 2013;2:82-90. PUBMED | CROSSREF

16. Van Gelder IC, Crijns HJ, Blanksma PK, et al. Time course of hemodynamic changes and improvement of exercise tolerance after cardioversion of chronic atrial fibrillation unassociated with cardiac valve disease. Am J Cardiol 1993;72:560-6. PUBMED | CROSSREF

17. Medi C, Kalman JM, Haqqani H, et al. Tachycardia-mediated cardiomyopathy secondary to focal atrial tachycardia: long-term outcome after catheter ablation. J Am Coll Cardiol 2009;53:1791-7. PUBMED | CROSSREF

18. Poutiainen AM, Koistinen MJ, Airaksinen KE, et al. Prevalence and natural course of ectopic atrial tachycardia. Eur Heart J1999;20:694-700.

PUBMED | CROSSREF

19. Meti N, Mongeon FP, Guerra PG, O'Meara E, Khairy P. Incessant atrioventricular nodal reentrant tachycardia with tachycardia-induced cardiomyopathy, biventricular thrombosis, and pulmonary emboli. HeartRhythm Case Rep 2016;2:142-5. PUBMED | CROSSREF

20. Selvaraj R, Ananthakrishnapillai A, Sadasivam R, Balachander J. "Pseudo PJRT"--fast-slow AV nodal reentrant tachycardia presenting with tachycardia-induced cardiomyopathy. Pacing Clin Electrophysiol 2013;36:e4-6. PUBMED | CROSSREF

21. Kim HS, Kim JS, Kwon SU, et al. A case of permanent junctional reciprocating tachycardia treated with radiofrequency catheter ablation. Korean Circ J2001;31:949-54. CROSSREF 
22. Aguinaga L, Primo J, Anguera I, et al. Long-term follow-up in patients with the permanent form of junctional reciprocating tachycardia treated with radiofrequency ablation. Pacing Clin Electrophysiol 1998;21:2073-8. PUBMED | CROSSREF

23. Cruz FE, Cheriex EC, Smeets JL, et al. Reversibility of tachycardia-induced cardiomyopathy after cure of incessant supraventricular tachycardia. J Am Coll Cardiol 1990;16:739-44. PUBMED | CROSSREF

24. Wang NC. Dual atrioventricular nodal nonreentrant tachycardia: a systematic review. Pacing Clin Electrophysiol 2011;34:1671-81. PUBMED | CROSSREF

25. Singh B, Kaul U, Talwar KK, Wasir HS. Reversibility of "tachycardia induced cardiomyopathy" following the cure of idiopathic left ventricular tachycardia using radiofrequency energy. Pacing Clin Electrophysiol 1996;19:1391-2. PUBMED | CROSSREF

26. Ryu K, Cho M, Choi D, et al. Recovery pattern and term of reversal of left ventricular remodeling in patients with congestive heart failure. Eur J Heart Fail Suppl 2006;5:146. CROSSREF

27. Donghua Z, Jian P, Zhongbo X, et al. Reversal of cardiomyopathy in patients with congestive heart failure secondary to tachycardia. J Interv Card Electrophysiol 2013;36:27-32. PUBMED | CROSSREF

28. Deshmukh PM, Krishnamani R, Romanyshyn M, Johnson AK, Noti JD. Association of angiotensin converting enzyme gene polymorphism with tachycardia cardiomyopathy. Int J Mol Med 2004;13:455-8. PUBMED | CROSSREF

29. Ryu KH, Tanaka N, Dalton N, et al. Force-frequency relations in the failing rabbit heart and responses to adrenergic stimulation. J Card Fail 1997;3:27-39. PUBMED | CROSSREF

30. Tomita M, Spinale FG, Crawford FA, Zile MR. Changes in left ventricular volume, mass, and function during the development and regression of supraventricular tachycardia-induced cardiomyopathy. Disparity between recovery of systolic versus diastolic function. Circulation 1991;83:635-44. PUBMED | CROSSREF

31. Spinale FG, Tomita M, Zellner JL, Cook JC, Crawford FA, Zile MR. Collagen remodeling and changes in LV function during development and recovery from supraventricular tachycardia. Am J Physiol 1991;261:H308-18. PUBMED | CROSSREF

32. Gupta S, Figueredo VM. Tachycardia mediated cardiomyopathy: pathophysiology, mechanisms, clinical features and management. Int J Cardiol 2014;172:40-6. PUBMED | CROSSREF

33. Kajstura J, Zhang X, Liu Y, et al. The cellular basis of pacing-induced dilated cardiomyopathy. Myocyte cell loss and myocyte cellular reactive hypertrophy. Circulation 1995;92:2306-17. PUBMED | CROSSREF

34. Zellner JL, Spinale FG, Eble DM, Hewett KW, Crawford FA Jr. Alterations in myocyte shape and basement membrane attachment with tachycardia-induced heart failure. Circ Res 1991;69:590-600. PUBMED | CROSSREF

35. Weber KT, Pick R, Silver MA, et al. Fibrillar collagen and remodeling of dilated canine left ventricle. Circulation 1990;82:1387-401. PUBMED | CROSSREF

36. Mukherjee R, Hewett KW, Spinale FG. Myocyte electrophysiological properties following the development of supraventricular tachycardia-induced cardiomyopathy. J Mol Cell Cardiol 1995;27:1333-48. PUBMED | CROSSREF

37. Balijepalli RC, Lokuta AJ, Maertz NA, et al. Depletion of T-tubules and specific subcellular changes in sarcolemmal proteins in tachycardia-induced heart failure. Cardiovasc Res 2003;59:67-77. PUBMED | CROSSREF

38. Ross J Jr, Miura T, Kambayashi M, Eising GP, Ryu KH. Adrenergic control of the force-frequency relation. Circulation 1995;92:2327-32. PUBMED | CROSSREF

39. O'Brien PJ, Ianuzzo CD, Moe GW, Stopps TP, Armstrong PW. Rapid ventricular pacing of dogs to heart failure: biochemical and physiological studies. Can J Physiol Pharmacol 1990;68:34-9. PUBMED | CROSSREF

40. Spinale FG, Grine RC, Tempel GE, Crawford FA, Zile MR. Alterations in the myocardial capillary vasculature accompany tachycardia-induced cardiomyopathy. Basic Res Cardiol 1992;87:65-79. PUBMED | CROSSREF 
41. Mueller KA, Heinzmann D, Klingel K, et al. Histopathological and immunological characteristics of tachycardia-induced cardiomyopathy. J Am Coll Cardiol 2017;69:2160-72.

PUBMED | CROSSREF

42. Lip GY, Heinzel FR, Gaita F, et al. European heart rhythm association/heart failure association joint consensus document on arrhythmias in heart failure, endorsed by the Heart rhythm society and the Asia Pacific heart rhythm society. Europace 2016;18:12-36.

PUBMED | CROSSREF

43. Gopinathannair R, Etheridge SP, Marchlinski FE, Spinale FG, Lakkireddy D, Olshansky B. Arrhythmiainduced cardiomyopathies: mechanisms, recognition, and management. J Am Coll Cardiol 2015;66:1714-28. PUBMED | CROSSREF

44. Ponikowski P, Voors AA, Anker SD, et al. 2016 ESC Guidelines for the diagnosis and treatment of acute and chronic heart failure: the Task Force for the diagnosis and treatment of acute and chronic heart failure of the European Society of Cardiology (ESC) developed with the special contribution of the Heart Failure Association (HFA) of the ESC. Eur Heart J 2016;37:2129-200. PUBMED | CROSSREF

45. Yancy CW, Jessup M, Bozkurt B, et al. 2013 ACCF/AHA guideline for the management of heart failure: executive summary: a report of the American College of Cardiology Foundation/American Heart Association Task Force on practice guidelines. Circulation 2013;128:1810-52.

PUBMED | CROSSREF

46. Nia AM, Gassanov N, Dahlem KM, et al. Diagnostic accuracy of NT-proBNP ratio (BNP-R) for early diagnosis of tachycardia-mediated cardiomyopathy: a pilot study. Clin Res Cardiol 2011;100:887-96. PUBMED | CROSSREF

47. Jeong YH, Choi KJ, Song JM, et al. Diagnostic approach and treatment strategy in tachycardia-induced cardiomyopathy. Clin Cardiol 2008;31:172-8.

PUBMED | CROSSREF

48. Hasdemir C, Yuksel A, Camli D, et al. Late gadolinium enhancement CMR in patients with tachycardiainduced cardiomyopathy caused by idiopathic ventricular arrhythmias. Pacing Clin Electrophysiol 2012;35:465-70. PUBMED | CROSSREF

49. Howard RJ, Stopps TP, Moe GW, Gotlieb A, Armstrong PW. Recovery from heart failure: structural and functional analysis in a canine model. Can J Physiol Pharmacol 1988;66:1505-12. PUBMED | CROSSREF

50. Dandamudi G, Rampurwala AY, Mahenthiran J, Miller JM, Das MK. Persistent left ventricular dilatation in tachycardia-induced cardiomyopathy patients after appropriate treatment and normalization of ejection fraction. Heart Rhythm 2008;5:1111-4.

PUBMED | CROSSREF

51. Nerheim P, Birger-Botkin S, Piracha L, Olshansky B. Heart failure and sudden death in patients with tachycardia-induced cardiomyopathy and recurrent tachycardia. Circulation 2004;110:247-52. PUBMED | CROSSREF

52. Boldt LH, Rolf S, Huemer M, et al. Optimal heart failure therapy and successful cardioversion in heart failure patients with atrial fibrillation. Am Heart J 2008;155:890-5.

PUBMED | CROSSREF

53. Van Gelder IC, Groenveld HF, Crijns HJ, et al. Lenient versus strict rate control in patients with atrial fibrillation. N Engl J Med 2010;362:1363-73. PUBMED | CROSSREF

54. Ling LH, Kalman JM, Ellims AH, et al. Diffuse ventricular fibrosis is a late outcome of tachycardiamediated cardiomyopathy after successful ablation. Circ Arrhythm Electrophysiol 2013;6:697-704. PUBMED | CROSSREF

55. Marrouche NF, Brachmann J, Andresen D, et al. Catheter ablation for atrial fibrillation with heart failure. N Engl J Med 2018;378:417-27.

PUBMED | CROSSREF 